\title{
Bedeutung internistischer Routineuntersuchungen bei Patienten mit obstruktivem Schlafapnoe-Syndrom
}

\author{
W. Galetke \\ W. Randerath \\ F. Feldmeyer \\ M. David \\ A. Trappe \\ F. Ingenabel
}

\author{
Importance of Routine Examinations in Patients with Obstructive \\ Sleep Apnea Syndrome
}

\section{Zusammenfassung}

Hintergrund und Fragestellung: Patienten mit obstruktivem Schlafapnoe-Syndrom (OSAS) weisen eine erhöhte Komorbidität an internistischen Erkrankungen wie arterieller Hypertonie, koronarer Herzkrankheit, chronisch-obstruktiver Bronchitis oder Stoffwechselkrankheiten auf. Wir analysierten prospektiv Häufigkeit und Konsequenzen pathologischer Befunde bei internistischen Zusatzuntersuchungen im Schlaflabor. Patienten und Methodik: Bei 250 Patienten mit OSAS wurden routinemäßig eine Lungenfunktion und Blutgasanalyse, ein Elektrokardiogramm (EKG) und Laboruntersuchungen (Blutbild, Leberwerte, Elektrolyte, Retentionsparameter, Blutzucker) durchgeführt. Zuvor erfolgte die Einschätzung, ob aufgrund von Anamnese und klinischem Befund diese Untersuchungen für indiziert gehalten wurden. Es wurden Häufigkeit und Art pathologischer Befunde sowie insbesondere die Konsequenzen, die aus diesen Ergebnissen gezogen wurden, analysiert. Ergebnisse: 129 Lungenfunktionsund Blutgasanalysen (51,6\% aller Untersuchungen) waren pathologisch, dabei wurden am häufigsten Hypoxämien (22\%) und obstruktive Ventilationsstörungen (16,4\%) festgestellt. Bei 19 Patienten (7,6\%) wurden Konsequenzen aus den Ergebnissen gezogen, davon war in 13 Fällen die Indikation vorher gesehen worden, d.h. sechs Ergebnisse (2,4\% aller Untersuchungen) waren Zufallsbefunde, die Konsequenzen nach sich zogen. Bei den Laborparametern waren 133 Ergebnisse pathologisch (53,2\% aller Untersuchungen), dabei dominierten erhöhte Nüchternglucosewerte $(26,8 \%)$ und Leberwerte $(20,4 \%)$. Konsequenzen erfolgten in 29 Fällen (11,6\%), davon waren 16 (6,4\%) als Zufallsbefund zu werten. 82 Elektrokardiogramme (32,8\% aller Untersuchungen) zeigten Auffälligkeiten, am häufigsten Hinweise für eine koronare Herzkrankheit (21,2\%). Bei fünf Patienten (2\%) resultierten
Abstract

Background and objective: In patients with obstructive sleep apnea syndrome (OSAS) there is an increased comorbidity of internal diseases such as hypertension, coronary heart disease, chronic obstructive pulmonary disease and endocrine diseases. We analyzed prospectively frequency and consequences of pathological results in routine examinations of internal medicine in sleep laboratory. Methods: 250 patients with OSAS underwent routine bodyplethysmography, blood- gas analysis, electrocardiogram and laboratory studies after anamnesis and clinical examination. Prior to this we indicated, whether we based on history and physical examination deemed any of these examinations necessary. Frequency and kind of pathological results as well as the consequences were analyzed. Results: 129 results of bodyplethysmography and blood-gas analysis were pathological (51.6\% of all investigations), most frequently hypoxemia (22\%) and obstructive pattern (16.4\%). Further steps were necessary in 19 patients (7.6\%); the indication was seen before in 13 patients, therefore 6 results with consequences (2.4\%) were not expected. Laboratory studies were abnormal in 133 patients $(53.2 \%$ of all investigations), most of them hyperglycaemia (26.8\%) and elevated liver enzymes (20.4\%). 29 results (11.6\%) had consequences, of which 16 (6.4\%) were not expected. 82 electrocardiograms were pathological (32.8\% of all investigations), presenting most frequently as coronary heart disease (21.2\%). Further steps were necessary in 5 patients ( $2 \%$, while that was not expected in 3 patients (1.2\%). Conclusions: Routine examinations of internal medicine in patients with OSAS frequently present pathological results, which seldom have further consequences. As most of the important results can be predicted by history and clinical investigation, pathological by chance diagnoses are rare. With the ex-

Institutsangaben

Klinik für Pneumologie, Allergologie und Schlafmedizin (Prof. Dr. K.-H. Rühle)

Universität Witten/Herdecke, Klinik Ambrock, Hagen

Widmung

Herrn Prof. Dr. Karl-Heinz Rühle zum 60. Geburtstag gewidmet

Korrespondenzadresse

Dr. med. W. Galetke · Klinik Ambrock · Ambrocker Weg 60 · 58091 Hagen ·E-mail: wohegal@t-online.de 
Konsequenzen, drei Befunde waren nicht erwartet worden (1,2\%). Folgerungen: Patienten mit OSAS weisen bei internistischen Zusatzuntersuchungen häufig pathologische Ergebnisse auf, aus denen sich jedoch nur in einem geringen Prozentsatz unmittelbare Konsequenzen ergeben. Die klinisch relevanten Befunde lassen sich durch Anamnese und körperlicher Untersuchung meistens vorhersagen, so dass pathologische Zufallsbefunde selten sind. Mit Ausnahme der Blutzuckerbestimmung sollte daher aus sozioökonomischen Gründen die routinemäßige Durchführung von Begleituntersuchungen bei OSAS-Patienten kritisch überdacht werden. ception of blood sugar tests routine examinations for patients with OSAS should be considered critically.

\section{Einleitung}

Patienten mit obstruktivem Schlafapnoe-Syndrom (OSAS) leiden häufig unter internistischen Begleiterkrankungen. Diese umfassen im Wesentlichen kardiovaskuläre und pulmonale Erkrankungen sowie Stoffwechselkrankheiten.

Die Prävalenz einer koronaren Herzkrankheit bei OSAS-Patienten wird mit bis zu 33\% angegeben [1,2], umgekehrt kann bei Patienten mit einer koronaren Herzkrankheit wesentlich häufiger ein OSAS nachgewiesen werden als in der Gesamtbevölkerung $[3,4]$. Die Angaben über das Vorliegen einer essentiellen arteriellen Hypertonie bei OSAS-Patienten schwanken zwischen $50 \%$ bis zu 90\% bei schwergradigen Befunden in der Polysomnographie [4-6]. Eine Assoziation mit einer chronisch-obstruktiven Atemwegserkrankung findet sich in 10-20\% der Patienten mit schlafbezogenen Atemregulationsstörungen [7-9], wobei diese signifikant niedrigere $\mathrm{PaO}_{2}$-Werte und höhere $\mathrm{PaCO}_{2}$-Werte am Tage sowie signifikant höhere pulmonal-arterielle Mitteldrücke als OSAS-Patienten ohne pulmonale Begleiterkrankung aufweisen $[10,11]$. In der Endokrinologie ist eine erhöhte Prävalenz des Schlafapnoe-Syndromes bei Patienten mit Diabetes mellitus, Hypothyreose oder Akromegalie festgestellt worden [12].

Daher werden bei Patienten mit obstruktivem Schlafapnoe-Syndrom zur Risikoabschätzung ergänzende apparative und laborchemische Untersuchungen wie z.B. Lungenfunktion, Blutgasanalyse, EKG und Langzeit-EKG oder ein Routinelabor empfohlen [13].

In diesem Zusammenhang interessierten uns die Häufigkeit pathologischer Befunde bei derartigen internistischen Zusatzuntersuchungen unter besonderer Berücksichtigung der aus ihnen gezogenen Konsequenzen und die klinische Vorhersagbarkeit pathologischer Ergebnisse.

\section{Patienten und Methodik}

In die vorliegende Studie wurden 250 konsekutive Patienten (59 Frauen, 191 Männer; Alter 54,5 \pm 11,3 Jahre; Body Mass Index $30 \pm 5,3 \mathrm{~kg} / \mathrm{m}^{2}$ ) eingeschlossen, bei denen wir während des stationären Aufenthaltes in unserem Schlaflabor ein obstruktives Schlafapnoe-Syndrom (OSAS) feststellten. Die Diagnose des OSAS wurde mittels einer nächtlichen Standard-Polysomnographie gestellt. Abgeleitet wurden dabei EEG, EOG, submentales EMG, EMG des M. tibialis, EKG, oronasaler Fluss (Thermistor), thorakale und abdominelle Effortsignale (Induktionsplethysmographie), Sauerstoffsättigung, Pulsfrequenz, Mikrophonregistrierung. Der mittlere Apnoe-Hypopnoe-Index (AHI) in dieser Gruppe betrug $30,2 \pm 19,6 /$ h.

Bei allen Patienten wurden vor der nächtlichen Messung eine Anamneseerhebung und klinische Untersuchung durchgeführt. Der/die Stationsarzt/ärztin war danach angehalten, anhand dieser Informationen zu entscheiden, ob ein Elektrokardiogramm, eine Lungenfunktionsanalyse und/oder klinische Laboruntersuchungen für erforderlich gehalten wurden.

Unabhängig von dieser Einschätzung erfolgte bei allen Patienten die Durchführung folgender Untersuchungen:

12-Kanal-Ruhe-EKG, Spirometrie und Ganzkörperplethysmographie, kapilläre Blutgasanalyse aus dem hyperämisierten Ohrläppchen, venöse Blutentnahme mit Bestimmung von kleinem Blutbild, Blutkörperchen-Senkungsgeschwindigkeit in der ersten Stunde, Natrium, Kalium, Kreatinin, Laktatdehydrogenase (LDH), Glutamat-Oxalazetat-Transaminase (GOT), Glutamat-PyruvatTransaminase (GPT), Gamma-Glutamyl-Transferase (GGT) sowie Nüchtern-Blutzuckerspiegel.

Im nächsten Schritt wurden von einem anderen Arzt, d.h. nicht vom zuständigen Stationsarzt die pathologischen Befunde und die aus ihnen gezogenen Konsequenzen analysiert. Hierbei wurden unabhängig von der klinischen Relevanz folgende Befunde als pathologisch angesehen:

EKG: Schenkelblockbilder, AV- bzw. SA-Blockierungen, Erregungsausbreitungsstörungen (terminal oder präterminal negative T-Zacke, horizontale oder dezendierende ST-Streckensenkungen), ventrikuläre bzw. supraventrikuläre Rhythmusstörungen, Myokardinfarktnarben ( $\mathrm{Q} \leq 0,04 \mathrm{~s}$ und $\geq 2 \mathrm{~mm}$ tief), Links- bzW. Rechtsherzhypertrophiezeichen.

Spirometrie bzw. Bodyplethysmographie: $\mathrm{FEV}_{1}<80$ Soll\%, $\mathrm{FEV}_{1} / \mathrm{VC}<80$ Soll\%, MEF 75/25<80 Soll\%, Raw $>0,3 \mathrm{kPa} / \mathrm{l} / \mathrm{s}$, VC $<80$ Soll\%, TLC $<80$ Soll\% bzW. $>120$ Soll\%, RV $>120$ Soll\%.

Blutgasanalyse: jede Abweichung des pO2, pCO2 oder des pHWertes vom altersentsprechenden Normwert.

Laborbefunde: jede Abweichung vom Normwert des jeweiligen Labors. 
Tab. 1 Anzahl und Art der pathologischen Befunde bei OSAS-Patienten ( $\mathrm{n}=250)$

\begin{tabular}{|c|c|c|c|c|c|}
\hline Lungenfunktion/Blutgasanalyse: & & Laborbefunde: & & EKG: & \\
\hline Hypoxämie & 55 & Blutzuckererhöhung & 67 & Erregungsausbreitungsstörungen & 37 \\
\hline nur periphere Obstruktion & 43 & Leberwerterhöhung & 51 & Schenkelblockbilder & 21 \\
\hline Lungenüberblähung & 32 & LDH-Erhöhung & 32 & Infarktnarben & 16 \\
\hline zentrale und periphere Obstruktion & 25 & Leukozytose & 10 & Vorhofflimmern & 7 \\
\hline nur zentrale Obstruktion & 16 & Erhöhung Retentionsparameter & 9 & ventrikuläre Extrasystolen & 6 \\
\hline Restriktion & 15 & Hyperkaliämie & 8 & Rechtsherzbelastung & 5 \\
\hline Hyperkapnie & 7 & Thrombozytopenie & 3 & AV-Block I. Grades & 4 \\
\hline \multirow[t]{5}{*}{ metabolische Azidose } & 1 & Hypernatriämie & 3 & linksventrikuläre Hypertrophie & 3 \\
\hline & & Anämie & 2 & & \\
\hline & & Leukozytopenie & 1 & & \\
\hline & & Hypokaliämie & 1 & & \\
\hline & & lipämisches Serum & 1 & & \\
\hline
\end{tabular}

Als Konsequenzen werteten wir Therapieempfehlungen, eine weitere stationäre oder ambulant empfohlene Diagnostik oder die Verlegung auf eine unserer Akutstationen, die aufgrund eines Untersuchungsbefundes ausgesprochen bzw. durchgeführt wurden.

\section{Statistik}

Es handelt sich um eine Querschnittsstudie. Die Zahlenangaben erfolgen als Absolutwerte oder prozentualer Anteil bezogen auf die Gesamtzahl der Untersuchungen.

\section{Ergebnisse}

Bei 42 der 250 Patienten (16,8\%) zeigten sämtliche Untersuchungsergebnisse Normalbefunde. 129 Lungenfunktions- bzw. Blutgasanalysen (51,6\%), 133 Laborbefunde $(53,2 \%)$ und 82 EKGs $(32,8 \%)$ waren pathologisch. Tab. 1 listet die pathologischen Befunde im Einzelnen auf.

Konsequenzen wurden bei 46 Patienten (18,4\%) insgesamt 53-mal gezogen, da bei sieben Patienten mehrere Untersuchungen Bedeutung erlangten. In 19 Fällen wurden Konsequenzen aus der Lungenfunktion/Blutgasanalyse gezogen, das entspricht 14,7\% der pathologischen Befunde und 7,6\% aller Untersuchungen. 29 Laborbefunde zogen Konsequenzen nach sich, entsprechend $21,8 \%$ der pathologischen und $11,6 \%$ der gesamten Untersuchungen. Die Anzahl der auffälligen Elektrokardiogramme mit Konsequenzen betrug fünf ( $6,1 \%$ aller pathologischen und $2 \%$ aller EKGs).

In der Tab. $\mathbf{2}$ werden die aus den Ergebnissen gezogenen Konsequenzen dargestellt.

In 13 der relevanten 19 Fällen (68,4\%), d.h. mit Konsequenzen belegten pathologischen Lungenfunktions- oder Blutgasergebnissen war vorher aufgrund der Aufnahmeuntersuchung die Indikation gesehen worden oder es handelte sich um bereits bekannte Befunde. Bei den Laborbefunden betrug dieser Anteil 13 der 29 relevanten Ergebnisse (44,8\%) und beim EKG zwei von fünf ( $40 \%)$.
Somit ergaben sich bei der Lungenfunktion bzw. Blutgasanalyse insgesamt sechs Zufallsbefunde, die Konsequenzen nach sich zogen $(2,4 \%$ aller Untersuchungen), bei den Laborbefunden 16 $(6,4 \%)$ und beim EKG drei $(1,2 \%)$.

Abb.1 gibt eine zusammenfassende Übersicht über die Anzahl der pathologischen Befunde, der daraus gezogenen Konsequenzen und der Zufallsbefunde.

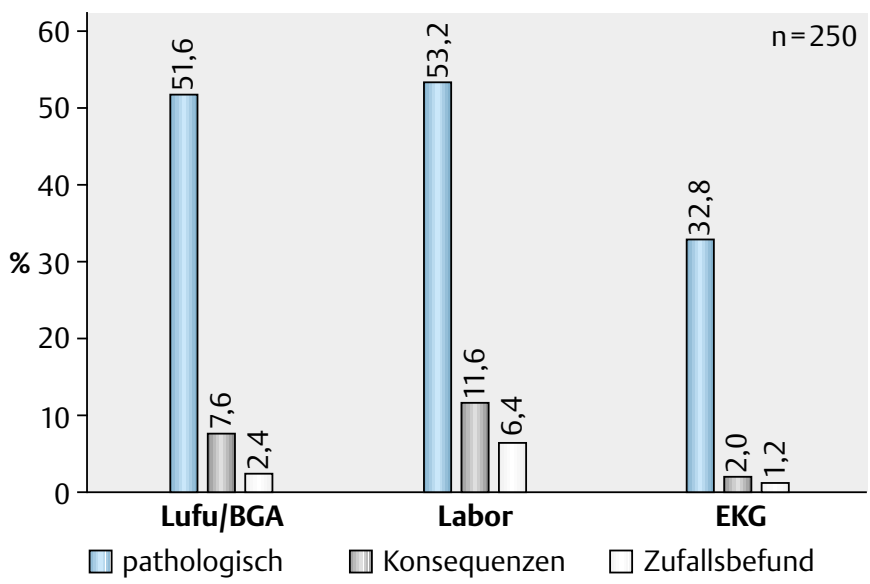

Abb. 1 Prozentualer Anteil der pathologischen Befunde, der pathologischen Befunde mit Konsequenzen und der Zufallsbefunde, d.h. der pathologischen Befunde, die durch Anamnese und klinische Untersuchung nicht erwartet wurden, an der Gesamtzahl der Untersuchungen. Lufu/BGA : Lungenfunktion/Blutgasanalyse

\section{Diskussion}

Zahlreiche Untersuchungen haben eine hohe Prävalenz kardiovaskulärer, pulmonaler sowie endokrinologischer Erkrankungen bei Patienten mit schlafbezogenen Atemregulationsstörungen nachgewiesen $[2-5,10,12,14-18]$. Aus diesem Grunde werden in vielen schlafmedizinischen Zentren routinemäßig internistische Zusatzuntersuchungen durchgeführt. So erhält in unserem Schlaflabor jeder Patient zumindest eine Lungenfunktion mit Blutgasanalyse, ein Ruhe-EKG sowie eine laborchemische Untersuchung, sofern diese nicht kurz zuvor ambulant erfolgt waren. Auch vor dem Hintergrund sozioökonomischer Überlegungen 
Tab. 2 Anzahl und Art der Konsequenzen bei OSAS-Patienten $(\mathrm{n}=250)$

\begin{tabular}{|c|c|c|c|c|c|}
\hline Lungenfunktion/Blutgasanalyse: & & Laborbefunde: & & EKG: & \\
\hline ambulante Abklärung/Kontrolle durch & & ambulante Kontrolle durch & & ambulante Abklärung durch & \\
\hline Pneumologen empfohlen & 9 & Hausarzt empfohlen & 15 & Kardiologen empfohlen & 3 \\
\hline Therapieempfehlung im Arztbericht & 6 & $\begin{array}{l}\text { ambulante Diagnostik bezüglich } \\
\text { Diabetes mellitus empfohlen }\end{array}$ & 14 & Verlegung auf Akutstation & 2 \\
\hline Verlegung auf Akutstation & 6 & Therapieempfehlung im Arztbericht & 2 & & \\
\hline
\end{tabular}

hinterfragten wir die Häufigkeit und insbesondere die Konsequenzen pathologischer Befunde bei diesen Untersuchungen.

Wir fanden bei etwas mehr als der Hälfte unseres Patientenkollektives unter Berücksichtigung der angegebenen Kriterien einen pathologischen Befund in der Lungenfunktion und/oder Blutgasanalyse, wobei obstruktive Ventilationsstörungen und Hypoxämien im Vordergrund standen. $16 \%$ der Patienten erfüllten die lungenfunktionsanalytischen Kriterien einer COPD [19-21]. Dies bestätigt die Ergebnisse früherer Untersuchungen kleinerer Patientenkollektive, die bei $11 \%$ bis $18 \%$ ihrer SBAS-Patienten eine obstruktive Ventilationsstörung dokumentierten $[9,11,14,22]$. Für dieses Patientenkollektiv wurde von Flenley der Begriff „Overlap-Syndrom“ geprägt [23]. Diese Patienten wiesen einen niedrigeren $\mathrm{PaO}_{2}$ und höheren $\mathrm{PaCO}_{2}$ am Tage sowie einen höheren pulmonal-arteriellen Mitteldruck als die SBAS-Patienten ohne begleitende COPD auf [11,15,24-26]. Dies erklärt auch den hohen Prozentsatz an Blutgasanalysen mit Hypoxämie bzw. Hyperkapnie in unserer Studie, der im Wesentlichen die Patienten mit einer obstruktiven Ventilationsstörung betraf.

Trotz der hohen Zahl an pathologischen Befunden in der Lungenfunktion/Blutgasanalyse wurden nur in knapp $8 \%$ aller Untersuchungen Konsequenzen gezogen. Das hängt vor allem damit zusammen, dass viele Patienten von niedergelassenen Pneumologen zugewiesen und somit bereits lungenfunktionsanalytisch untersucht sowie entsprechend behandelt wurden. Pathologische Ergebnisse wurden bei diesen Patienten zwar von uns registriert, jedoch nur bei Abweichungen vom Vorbefund oder bei offensichtlich inadäquater Therapie mit Konsequenzen belegt.

Relevante „Zufallsbefunde“, d.h. pathologische Ergebnisse, die vorher nicht bekannt und bei der Aufnahmeuntersuchung nicht vermutet worden waren, blieben daher mit 2,4\% aller Untersuchungen sehr selten. Insbesondere war bei jedem der sechs Patienten, die aufgrund der Lungenfunktion/Blutgasanalyse auf unsere Akutstation verlegt wurden, vorher die Indikation zu diesen Untersuchungen gesehen worden.

Der hohe Anteil an pathologischen Laborwerten resultierte im Wesentlichen aus erhöhten Nüchternglucose-Werten und Transaminasen. Dies ist insbesondere hinsichtlich der Blutzuckerwerte nicht überraschend, da seit Jahren nicht nur eine aufgrund gemeinsamer Risikofaktoren erhöhte Komorbidität zwischen Diabetes mellitus und SBAS bekannt ist $[27,28]$, sondern auch ein pathogenetischer Zusammenhang zwischen beiden Erkrankungen diskutiert wird. Mehrere Studien konnten eine Korrelation zwischen der Anzahl der Apnoen/Hypopnoen und dem Plasmainsulin- bzw. Glucosespiegel belegen, so dass nächtliche Ap- noen über die vermehrte Ausschüttung von Katecholaminen und Kortisol eine Hyperinsulinämie sowie Insulinresistenz induzieren und somit zum Diabetes mellitus führen könnten $[12,18,29]$. Kiely et al. dokumentierten bei $16 \%$ ihrer OSAS-Patienten eine gestörte Glucosetoleranz bzw. einen manifesten Diabetes mellitus, wobei dies bei etwa der Hälfte der Betroffenen nicht bekannt war [17]. Levinson et al. fanden sogar bei $30 \%$ ihres Patientenkollektives eine gestörte Glucosetoleranz [5]. In unserer Studie lag der Anteil der Patienten mit erhöhten Nüchternglucose-Werten mit $26 \%$ vergleichbar hoch, wobei wir im Gegensatz zu den vorgenannten Arbeiten keinen oralen Glukosetoleranztest durchführten. Auffallend war auch hier, dass bei $5 \%$ aller Patienten eine diabetische Stoffwechsellage nicht bekannt war und demzufolge eine weitere Abklärung als Konsequenz resultierte. Dies erklärt auch den insgesamt höheren Anteil an Konsequenzen bzw. pathologischen Zufallsbefunden bei den Laborwerten im Vergleich zu den anderen Untersuchungen.

Immerhin 20\% aller Patienten wiesen erhöhte Leberwerte auf, wobei maximal ein Anstieg auf das Vierfache der Norm beobachtet wurde. Als Erklärung ist einerseits bei einem Teil dieser Patienten der anamnestisch angegebene Alkoholkonsum zu diskutieren, andererseits der erhöhte BMI in der Studiengruppe zu berücksichtigen. Frühere Studien wiesen bereits eine Assoziation zwischen BMI und erhöhten Leberenzymen unabhängig vom Alkoholkonsum bei ansonsten Gesunden nach [30-34]. Neben den erhöhten Glucose- und Leberwerten verblieben lediglich drei weitere als Zufallsbefund imponierende Laborwerte. Hierbei handelte es sich um zwei beschleunigte Blutkörperchen-Senkungsgeschwindigkeiten sowie einen diskret erhöhten Kreatininwert.

Ein Drittel der in unserer Studie durchgeführten Elektrokardiogramme zeigte einen pathologischen Befund. Dabei dominierten Erregungsausbreitungsstörungen, die sich bei 15\% der Patienten fanden. Dies entspricht den Ergebnissen einer früheren Studie, die in einem kleineren Patientenkollektiv bei 14,5\% der Elektrokardiogramme Ischämiezeichen dokumentierte [35]. Berücksichtigt man zudem die 16 Patienten, die nach elektrokardiographischen Kriterien einen Myokardinfarkt durchgemacht hatten, so wäre aufgrund des EKG eine KHK bei $21 \%$ unserer Patienten zu vermuten. In einer anderen Untersuchung gelang der angiographische Nachweis einer koronaren Herzkrankheit sogar bei etwa $25 \%$ der Patienten mit einer obstruktiven Schlafapnoe [2].

Lediglich 5\% unserer Patienten wiesen Rhythmusstörungen im Ruhe-EKG auf. Unsere Ergebnisse stützen somit eher die Beobachtungen früherer Untersuchungen, dass bei OSAS-Patienten wesentlich häufiger nächtliche Arrhythmien festgestellt werden 
können und eine Vorhersage dieser Ereignisse aufgrund des Ruhe-EKG nicht möglich ist $[16,36]$.

Konsequenzen wurden in unserer Studie nur aus fünf pathologischen EKG-Befunden gezogen. Das hängt zum einen wiederum damit zusammen, dass bei einem Großteil der Patienten die Befunde bereits durch Voruntersuchungen bekannt waren und zum anderen viele Befunde in den Kontext anderer bekannter Erkrankungen wie arterieller Hypertonie oder chronisch-obstruktiver Bronchitis gestellt werden konnten. Daher ergaben sich lediglich drei pathologische Zufallsbefunde, deren geringe klinische Relevanz aber nur eine weitere ambulante Abklärung als Empfehlung zur Folge hatten. Bei den zwei Patienten, die aufgrund einer bislang nicht bekannten Tachyarrhythmia absoluta auf unsere Akutstation verlegt werden mussten, war die Indikation zur Anfertigung eines EKGs schon bei der klinischen Untersuchung aufgefallen.

Einschränkend muss bei den dargestellten Ergebnissen berücksichtigt werden, dass durch die ärztlichen Mitarbeiter im Schlaflabor in Kenntnis der laufenden Studie möglicherweise auch solchen Befunden Bedeutung beigemessen wurde, die sonst für irrelevant gehalten worden wären und somit die Anzahl der Konsequenzen im klinischen Alltag sogar noch niedriger anzunehmen ist. Weiterhin bleibt anzumerken, dass von einem Teil der Patienten die bereits ambulant erhobenen Befunde der Klinik zur Verfügung gestellt worden waren, so dass hier eine erneute Untersuchung im klinischen Alltag nicht erforderlich gewesen wäre.

Zusammenfassend fanden wir bei OSAS-Patienten eine hohe Anzahl pathologischer Befunde bei internistischen Routineuntersuchungen und konnten somit die Komorbidität zwischen OSAS und kardiovaskulären, pulmonalen und endokrinologischen Erkrankungen bestätigen. Unmittelbare Konsequenzen wurden jedoch nur in einem geringen Prozentsatz aus diesen Befunden gezogen, da die Begleiterkrankungen bereits bekannt oder die Befunde von geringer klinischer Bedeutung waren. Während die meisten relevanten Befunde in der Lungenfunktion/Blutgasanalyse und im EKG bereits klinisch vermutet worden waren, ergaben sich bei den Laborparametern durch die große Anzahl erhöhter Blutzuckerwerte häufiger pathologische Zufallsbefunde. Daher erscheint eine routinemäßige Blutzuckerbestimmung bei OSAS-Patienten sinnvoll. Die Bestimmung anderer Laborparameter und die Durchführung von Lungenfunktion/Blutgasanalyse und EKG sollten dagegen auch unter sozioökonomischen Gesichtspunkten nur dann erfolgen, wenn konkrete Hinweise auf eine Veränderung oder Störung im entsprechenden Organsystem vorhanden sind.

\section{Literatur}

${ }^{1}$ Konermann M, Sanner B, Sturm A. Schlafbezogene Atmungsstörungen und assoziierte kardiovaskuläre Erkrankungen. Med Klin 1995; 90: $480-485$

2 Sanner B, Sturm A, Konermann M. Koronare Herzerkrankung bei Patienten mit obstruktiver Schlafapnoe. Dtsch Med Wschr 1996; 121: $931-935$

${ }^{3}$ Peker Y, Kraiczi H, Hedner J et al. An independent association between obstructive sleep apnoea and coronary artery disease. Eur Respir J 1999; 13: 179-184

${ }^{4}$ Sturm A, Sanner B. Schlafmedizin. Ein neues Querschnittsfach der Inneren Medizin. Dtsch Med Wschr 1999; 124: 1573-1576

${ }^{5}$ Levinson PD, McGarvey ST, Carlisle CC et al. Adiposity and cardiovascular risk factors in men with obstructive sleep apnea. Chest 1993; 103: $1336-1342$

${ }^{6}$ Mayer J, Becker H, Köhler U et al. Variabilität von arteriellem Blutdruck und Herzfrequenz bei Schlafapnoe. Prax Klin Pneumol 1987; 41: $385-386$

${ }^{7}$ Fletcher EC. Chronic lung disease in the sleep apnea syndrome. Lung 1990; 168 (suppl): 751 - 761

${ }^{8}$ Stacchi R, Weiss M, Brändli O. Lässt sich das obstruktive Schlafapnoesyndrom aufgrund von Anamnese und klinischen Untersuchungsbefunden voraussagen? Schweizerische Rundschau für Medizin (Praxis) 1995; 9: 247-254

${ }^{9}$ Weitzenblum E, Krieger J, Oswald M et al. Chronic obstructive pulmonary disease and sleep apnea syndrome. Sleep 1992; 15: S33-S35

${ }^{10}$ Fletcher EC, Schaaf JW, Miller J et al. Long-term cardiopulmonary sequelae in patients with sleep apnea and chronic lung disease. Am Rev Respir Dis 1987; 135: 525-533

${ }^{11}$ Orth M, Rasche K, Bauer TT et al. Häufigkeit der chronisch-obstruktiven Atemwegserkrankung bei Patienten mit obstruktivem Schlafapnoesyndrom. Pneumologie 1996; 50: 286-289

12 Rosenow F, McCarthy V, Caruso AC. Sleep apnoea in endocrine disease. J Sleep Res 1998; 7: 3-11

${ }^{13}$ Hein H, Raschke F, Köhler D et al. Leitlinie zur Diagnostik und Therapie schlafbezogener Atmungsstörungen beim Erwachsenen. Pneumologie 2001; 55: 339-342

${ }^{14}$ Chaouat A, Weitzenblum E, Krieger J et al. Association of chronic obstructive pulmonary disease and sleep apnea syndrome. Am J Respir Care Med 1995; 151: $82-86$

${ }^{15}$ Chaouat A, Weitzenblum E, Krieger J et al. Prognostic value of lung function and pulmonary haemodynamics in OSA patients treated with CPAP. Eur Respir J 1999; 13: 1091 - 1096

${ }^{16}$ Fett I, Lübbeke A, Hay J et al. Kardiopulmonale Risikofaktoren bei Schlafapnoepatienten. Pneumologie 1991; 45: 265-270

17 Kiely JL, McNicholas WT. Cardiovascular risk factors in patients with obstructive sleep apnoea syndrome. Eur Respir J 2000; 16: 128-133

${ }^{18}$ Strohl KP. Diabetes and sleep apnea. Sleep 1996; 19 (10): S225 - S228

19 The COPD Guideline Group of the Standards of Care Committee of the BTS. BTS guidelines for the management of chronic obstructive pulmonary disease. Thorax 1997; 52 (suppl 5): S1 -S28

${ }^{20}$ Siafakas NM, Vermeire P, Pride NB et al. Optimal assessment and management of chronic obstructive pulmonary disease (COPD). ERS-Consensus Statement. Eur Respir J 1995; 8: 1398 - 1420

${ }^{21}$ American Thoracic Society. Standards for the diagnosis and care of patients with chronic obstructive pulmonary disease. Am J Respir Crit Care Med 1995; 152 (suppl): S77-S121

${ }^{22}$ Fischer J, Raschke F. Häufigkeit eines obstruktiven Schlafapnoesyndroms in Kombination mit einer chronisch obstruktiven Atemwegserkrankung. Pneumologie 1993; 47: $731-734$

${ }^{23}$ Flenley DC. Sleep in chronic obstructive pulmonary disease. Clin Chest Med 1985; 328: $537-599$

${ }^{24}$ Bradley TD, Rutherford R, Grossman RF et al. Role of daytime hypoxemia in the pathogenesis of right heart failure in the obstructive sleep apnea syndrome. Am Rev Respir Dis 1985; 131: 835-839

25 Douglas NJ. Are sleep studies necessary in COPD? Lung 1990; Suppl: 943-947

${ }^{26}$ Shu Chan C, Grunstein RR, Bye PTP et al. Obstructive sleep apnea with severe chronic airflow limitation. Comparison of hypercapnic and eucapnic patients. Am Rev Respir Dis 1989; 140: 1274-1278

${ }^{27}$ Charoenpan P, Thanakitcharu S, Muntarbhorn K et al. Sleep apnoea syndrome in Ramathibodi Hospital: Clinical and polysomnographic baseline data. Respirology 1999; 4: 371-374 
${ }^{28}$ Ficker JH, Dertinger SH, Siegfried W et al. Obstructive sleep apnoea and diabetes mellitus: the role of cardio-vascular autonomic neuropathy. Eur Respir J 1998; 11: 14- 19

${ }^{29}$ Elmasry A, Lindberg E, Berne $\mathrm{C}$ et al. Sleep-disordered breathing and glucose metabolism in hypertensive men: a population-based study. J Intern Med 2001; 249 (2): 153 - 161

30 Bellentani S, Saccoccio G, Masutti F et al. Prevalence of and risk factors for hepatic steatosis in northern Italy. Ann Intern Med 2000; 132 (2): $112-117$

${ }^{31}$ Burns CJ, Boswell JM, Olsen GW. Liver enzyme activity and body mass index. J Occup Environ Med 1996; 38 (12): 1248-1252

32 Guzzaloni G, Grugni G, Minocci A et al. Liver steatosis in juvenile obesity: correlations with lipid profile, hepatic biochemical parameters and glycemic and insulinemic responses to an oral glucose tolerance test. Int J Obes Relat Metab Disord 2000; 24 (6): 772 -776

${ }^{33}$ Luyckx FH, Desaive C, Thiry A et al. Liver abnormalities in severely obese subjects: effect of drastic weight loss after gastroplasty. Int Obes Metab Disord 1998; 22 (3): 222-226

${ }^{34}$ Marchesini G, Brizi M, Morselli-Labate AM et al. Association of nonalcoholic fatty liver disease with insulin resistance. Am J Med 1999; 107 (5): $450-455$

35 Paiva T, Vasconcelos P, Leitão AN et al. Obstructive sleep apnea. Clinical and laboratory studies. Acta Medica Portuguesa 1994; 7: 43-50

${ }^{36}$ Köhler U, Becker H, Borrmann R et al. Das EKG bei Patienten mit schlafbezogenen Atemregulationsstörungen - Seine Stellung in Diagnostik und Therapie. Prax Klin Pneumol 1987; 41: 380- 384

\section{Preisausschreibungen}

\section{Roger-Bone-Preis}

Der Preis wird ausgeschrieben von der Deutschen Sepsis-Gesellschaft e.V., Dotation 3000 Euro, gestiftet von der Lilly Deutschland $\mathrm{GmbH}$ (Bad Homburg) zur Förderung von Publikationen auf dem Gebiet der klinischen Sepsisforschung.

Der Preis wird an Wissenschaftler aus dem deutschsprachigen Raum anlässlich des 6. Kongresses der Deutschen Interdisziplinären Vereinigung für Intensivmedizin (DIVI) am 15. 11. 2002 in Hamburg verliehen. Bewerbungen mit in den Jahren 2001 oder 2002 publizierten oder zur Publikation angenommenen Arbeiten können bis spätestens 30. 9. 2002 eingereicht werden an:

Prof. Dr. Konrad Reinhart, Vorsitzender der Deutschen Sepsis, Gesellschaft e.V., Universitätsklinikum Jena, Klinik für Anästhesiologie und Intensivtherapie, Bachstr. 18, 07740 Jena.

\section{Hugo-Schottmüller-Preis}

Der Preis wird ausgeschrieben von der Deutschen Sepsis-Gesellschaft e.V., Dotation 3000 Euro, gestiftet von der SIRS-Lab GmbH (Jena) zur Förderung von Habilitationsschriften auf dem Gebiet der Grundlagenforschung der Sepsis. Der Preis wird an Wissenschaftler aus dem deutschsprachigen Raum anlässlich des 6 . Kongresses der Deutschen Interdisziplinären Vereinigung für Intensivmedizin (DIVI) am 15. 11. 2002 in Hamburg verliehen. Bewerbungen mit in den Jahren 2001 oder 2002 publizierten oder zur Publikation angenommenen Arbeiten können bis spätestens 30. 9. 2002 eingereicht werden an:

Prof. Dr. Konrad Reinhart, Vorsitzender der Deutschen Sepsis, Gesellschaft e.V., Universitätsklinikum Jena, Klinik für Anästhesiologie und Intensivtherapie, Bachstr. 18, 07740 Jena. 